\title{
A Belief Rule based Expert System to Diagnose Dengue Fever under Uncertainty
}

\author{
Mohammad Shahadat Hossain and Israt Binteh Habib \\ Department of Computer Science and Engineering \\ University of Chittagong \\ University-4331, Bangladesh \\ hossain_ms@cu.ac.bd, isratjahan15.cse@gmail.com
}

\author{
Karl Andersson \\ Pervasive and Mobile Computing Laboratory \\ Luleå University of Technology \\ SE-931 87 Skellefteå, Sweden \\ karl.andersson@1tu.se
}

\begin{abstract}
Dengue Fever is a debilitating mosquito-borne disease, causing sudden fever, leading to fatality in many cases. A Dengue patient is diagnosed by the physicians by looking at the various signs, symptoms and risk factors of this disease. However, these signs, symptoms and the risk factors cannot be measured with $100 \%$ certainty since various types of uncertainties such as imprecision, vagueness, ambiguity, and ignorance are associated with them. Hence, it is difficult for the physicians to diagnose the dengue patient accurately since they don't consider the uncertainties as mentioned. Therefore, this paper presents the design, development and applications of an expert system by incorporating belief rule base as the knowledge representation schema as well as the evidential reasoning as the inference mechanism with the capability of handling various types of uncertainties to diagnose dengue fever. The results generated from the expert system are more reliable than from fuzzy rule based system or from human expert.
\end{abstract}

Keywords-Belief Rule Base; Dengue Fever; Evidential Reasoning; Expert System; Uncertainty

\section{INTRODUCTION}

Dengue is a mosquito-borne viral disease; causing flu-like illness which can be turned into lethal complication, called severe dengue. The global occurrence of this disease has increased dramatically in the recent years, and hence; half of the world's population is now at risk [1]. Approximately 975 million of these populations live in urban areas in tropical and sub-tropical countries of South-East Asia, the Pacific and the Americas [2]. Ninety percent of the dengue patients are children under the age of 15 years and it is endemic in 112 countries [3].

The dengue virus usually spreads through a cycle of transmission, comprising human-to-mosquito-to-human [4][5]. Typically, a high level of dengue virus in the blood of a person is noticed four days after being bite by an infected Aedes aegypti mosquito. While, five days after being bite by the mosquito, the various signs and symptoms of dengue fever, consisting of fever, headache, rash, congested conjunctive and relative bradicardia are noticed [6]. Although there is no specific treatment for dengue or severe dengue, an early diagnosis by looking at the signs and symptoms could play an important role to reduce the rate of mortality [1].

There are three different forms of dengue fever. They are Dengue Fever (DF), Dengue Haemorrhagic Fever (DHF) and Dengue Shock Syndrome (DSS). DF is characterized by high fever (usually between $39.5-41{ }^{\circ} \mathrm{C}$, persisting for $1-7$ days only), severe headache, pain behind eyes, congested conjunctiva, muscle and joint pains, nausea or vomiting and rash [7]. While DHF is a less common but it is a severe form of dengue where the patient shows bleeding. DSS is considered as the most risky, responsible for most of the deaths, especially in children. It is important to note that all of the signs and symptoms may not appear concurrently, making the diagnosis of dengue difficult. In addition to the signs and symptoms, various risk factors such as mosquito breeding place, mosquito bite and population under risk of dengue fever should be taking into account to perform the task of diagnosis of the disease.

However, the accurate measurement of these signs, symptoms and risk factors is challenging because there exists different types of uncertainties with each of them. Eventually, it is difficult to obtain the accurate early diagnostics result, which is crucial to detect this disease, and to safe human life, especially the children as mentioned. Therefore, the research presented in this paper carried out a comprehensive survey in consultation with the expert physicians, located in the various hospitals, medical colleges in the City of Chittagong, Bangladesh to identify the various types of uncertainties associated with each sign, symptom and risk factor. It is important to note that dengue frequently occurs in this part of Bangladesh. Table 1 illustrates the results of the survey, for example, the types of uncertainties associated with "fever" as identified, consisting of randomness and ignorance. The reason for this is that during dengue fever, temperature may go up and down, which may cause uncertainty due to randomness and ignorance, resulting wrong diagnosis of the disease.

The current gold standard technique in monitoring risk in dengue is to take the patient's blood sample for the measurement of total haematocrit (HCT), haemoglobin $(\mathrm{Hb})$ concentration, platelet count, and liver function status [2][8]. However, such approach is considered as invasive and hence, regular blood sampling will cause further injury to the subcutaneous tissue and potential risk to the dengue patients [9]. On the contrary, non-invasive approach to diagnose dengue fever is in practice, carried out by looking at the signs, symptoms and risk factors. However, the effectiveness and the accuracy of the non-invasive approach depend on handling the various types of uncertainties. Therefore, the traditional way of diagnosis of dengue fever by using non-invasive approach, usually carried out by the physicians, is incapable of producing accurate result. This can be addressed by developing an expert 
system with the capability of handling uncertainty in knowledge representation as well as in inference mechanism. Therefore, this paper presents the development of an expert system (ES), based on a methodology, known as the belief rule-base inference methodology using the evidential reasoning (RIMER) [10].

TABLE I. TyPES OF UNCERTAINTY IN DENGUE DisEASE

\begin{tabular}{|c|c|c|}
\hline $\begin{array}{l}\text { Dengue } \\
\text { Factors }\end{array}$ & Uncertainty Types & Discussion \\
\hline Fever & $\begin{array}{l}\text { Ignorance, } \\
\text { randomness }\end{array}$ & $\begin{array}{l}\text { There are many similarities among } \\
\text { dengue, chikungunya and flu virus } \\
\text { [10]. In dengue disease temperature } \\
\text { goes ups and down, so random data } \\
\text { may give wrong result. }\end{array}$ \\
\hline Headache & $\begin{array}{l}\text { Imprecision, } \\
\text { incompleteness }\end{array}$ & $\begin{array}{l}\text { Patients sometimes may be unable } \\
\text { to express their pain precisely. } \\
\text { Without precise expression expert } \\
\text { assessment is incomplete. }\end{array}$ \\
\hline Rash & Ignorance & $\begin{array}{l}\text { Patients may have some rash in their } \\
\text { body but they may ignore this } \\
\text { symptom. }\end{array}$ \\
\hline $\begin{array}{l}\text { Congested } \\
\text { Conjunctiva }\end{array}$ & Inconsistency & $\begin{array}{l}\text { It is possible that patients may have } \\
\text { allergic problem. That's why } \\
\text { conjunctivitis occurs. In this way } \\
\text { inconsistent data may be mixed with } \\
\text { dengue assessment. }\end{array}$ \\
\hline $\begin{array}{l}\text { Relative } \\
\text { bradicardia }\end{array}$ & $\begin{array}{l}\text { Complexity, } \\
\text { inconsistency }\end{array}$ & $\begin{array}{l}\text { As relative bradicardia is measured } \\
\text { by digital blood pressure machine, it } \\
\text { contains quantitative data. So } \\
\text { complex information may lead to } \\
\text { misdiagnosis. }\end{array}$ \\
\hline $\begin{array}{l}\text { Number of } \\
\text { mosquito bite }\end{array}$ & $\begin{array}{l}\text { Vagueness, } \\
\text { ignorance, } \\
\text { randomness }\end{array}$ & $\begin{array}{l}\text { Expert avoids measuring this issue. } \\
\text { So ignorance occurs. Sometimes } \\
\text { mosquito bite is low but patients } \\
\text { may have dengue fever, sometimes } \\
\text { mosquito bite is high but patients } \\
\text { may don't have dengue fever } \\
\text { consequently. So random data may } \\
\text { lead to misdiagnosis. }\end{array}$ \\
\hline $\begin{array}{l}\text { Mosquito } \\
\text { breeding place }\end{array}$ & Incompleteness & Expert assessment is incomplete. \\
\hline Population & Ignorance & $\begin{array}{l}\text { Experts may not have the correct } \\
\text { information about the population } \\
\text { density of the area. }\end{array}$ \\
\hline
\end{tabular}

The remaining of the paper is structured as follows. Section two presents the related works. Section three provides an overview of RIMER methodology. Section four presents the architecture, design and implementation of the proposed BRBES. Experimental results and discussion are then presented. A conclusion is included to summaries the contribution of the research.

\section{LITERURE REVIEW}

Fuzzy logic based expert system to diagnose seven tropical infectious diseases developed [11], where dengue fever considered. This system used fuzzy logic based knowledge representation schema to handle uncertainty due to vagueness while certainty factor used to handle the inability of an expert in defining the relationship between the symptoms of the disease with certainty. Artificial Neural Network (ANN) based system proposed to facilitate the analysis of dengue fever [12]. It describes a non-invasive prediction system, enabling the prediction of the day of the defervescence of the dengue patient. This system used multilayer feed-forward neural networks and used clinical signs and symptoms to carry out the prediction. A rule based expert system to classify risk in dengue infections by using bioelectrical impedance analysis developed [13]. An expert system for diagnosing dengue fever based on basic information gathered from literature study and expert interview developed [14]. Although fuzzy logic based expert system can handle uncertainty due to vagueness, ambiguity and fuzziness, it is incapable of handling uncertainty due to ignorance, incompleteness and randomness. From Table 1 it can be observed that the uncertainties associated with the signs and symptoms of the dengue fever are associated with incompleteness, ignorance and randomness in addition to the vagueness, fuzziness and ambiguity. ANN based approach is a type of black-box based system, which is incapable of handling various types of uncertainties associated with the signs and symptoms of dengue fever. Neither [13] nor [14] used the rule base in the knowledge representation schema or in the inference mechanism are capable of addressing the uncertainty issues that have been elaborated in related to dengue fever.

However, Belief Rule Based Expert Systems (BRBES) are widely used in the clinical domain where there exists plethora of uncertain clinical information [15][16]. In the BRBES, belief rule base (BRB) is used to develop the knowledge base by addressing various types of uncertainties such as incompleteness, ambiguity, vagueness and imprecision. While inference mechanism consists of number procedures namely input transformation, rule activation weight calculation, belief update and rule aggregation by using evidential reasoning algorithm. The strength of BRBES's inference procedure is that it has the capability to handle various types of uncertainty and hence, facilitates a robust decision making process. In the following section an overview of BRBES methodology will be presented.

\section{OVERVIEW OF BRBESS METHODOLOGY}

The Belief Rule Based Expert Systems (BRBESs) methodology consists of two parts, namely knowledge representation schema by using belief rule base and the inference mechanism, which consists of a number of procedures. This section elaborates each of them.

\section{A. Domain Knowledge Representation using BRB}

Belief Rule Base (BRB) is the extension of traditional IFTHEN rule base where belief degrees are embedded with the referential values of the consequent attribute while antecedent part contains the referential value of the antecedent attribute. A belief rule base (BRB) is used to capture the nonlinear causal relationships under uncertainty, which is not the case with the traditional IF-THEN rule base. Equation (1) represents the example of a belief rule.

$$
\begin{gathered}
R_{k}:\left\{\begin{array}{c}
\operatorname{IF}\left(X_{1} \text { is } A_{1}^{k}\right) \Lambda\left(X_{2} \text { is } A_{2}^{k}\right) \Lambda \ldots \ldots . \Lambda\left(X_{T_{k}} \text { is } A_{T_{k}}^{k}\right) \\
\operatorname{THEN}\left\{\left(D_{1} i s B_{1 k}\right),\left(D_{2} i s B_{2 k}\right), \ldots \ldots .,\left(D_{N} i s B_{N k}\right)\right\}
\end{array}\right. \\
\sum_{j=1}^{N} \beta_{j k} \leq 1
\end{gathered}
$$

with a rule weight $\theta_{k}(k=1, \ldots, L)$

and attributes weights $\delta_{1}, \delta_{2}, \ldots, \delta_{T_{k}}$

Here, $X_{i_{k}}\left(i=1, \ldots, T_{k}\right)$ is referred to as $i^{\text {th }}$ antecedent attribute while $A_{T_{k}}^{k}\left(i=1,2, \ldots, T_{k}\right)$ represents its referential 
value. $T_{k}$ is the number of antecedent attributes used in the kth rule. $D_{\mathrm{i}}(i=1,2, \ldots, N)$ is the referential value of the consequent attribute with degree of belief $\beta_{\mathrm{ik}}(i=1,2, \ldots, N)$. The $\mathrm{k}^{\text {th }}$ rule is said to be complete if $\sum_{j=1}^{n} \beta_{j k}=1$ otherwise, it is incomplete. $L$ is the number of belief rules in the rule base. Equation (2) represents the more precise example of a belief rule from the domain of dengue fever.

$$
R_{1}:\left\{\begin{array}{c}
\text { IF (Population is High }) \cap(\text { Mosquito Breeding Place is } \\
\text { Medium }) \cap(\text { Mosquito Bite is Medium }) \\
\text { THEN Dengue Risk Assessment is } \\
\{(\text { High }(0.4), \text { Medium }(0.6), \operatorname{Low}(0.2))\}
\end{array}\right.
$$

Here, high, medium and low are the referential values of the consequent attribute "Dengue Risk Assessment" of the belief rule. The above rule states that the risk of dengue of a person in his present living condition is high with $40 \%$ degree of belief, while medium with $60 \%$ degree of belief and low with $0 \%$ degree of belief. This belief rule is complete since the summation of degree of belief associated with each of the referential value of the consequent attribute is 1 $[(0.4+0.6+0+0)=1]$. Hence, it can be argued that the rule is complete and in this way uncertainty due to incompleteness can be addressed in the belief rule base.

\section{B. Inference Procedure in BRBESs}

The inference procedure of BRBEs consists of input transformation, rule activation weight calculation, belief update and rule aggregation as will be discussed below.

\section{1) Input Transformation}

The input transformation consists of finding the matching degrees of the input value of an antecedent attribute to its various referential values by using equations (3), (4) and (5). Hence, this is equivalent to transforming an input value into a distribution over the referential values of an antecedent attribute. The input value of an antecedent attribute (for example headache) is usually collected from the patients or from the physicians in terms of linguistic terms such as high, medium, low and normal. It can also be collected as numerical value for example in case of fever.

$$
\begin{gathered}
I F(H \text { value } \geq \text { Input value } \geq M \text { value }) \text { THEN } \\
\text { Medium }=\frac{H \text { value }- \text { Input value }}{H \text { value }-M \text { value }}, \text { High }=1-\text { Medium, Low }=0.0 \\
\text { Medium }=\frac{\text { Mvalue }- \text { Inputvalue }}{M \text { value }- \text { Lvalue }}, \text { Medium }=1-\text { Low High }=0.0
\end{gathered}
$$

Table 2 shows the matching degree of the antecedent attribute value into its different referential values. For example, the input value of the antecedent attribute "Rash" is identified as "low", which is weighted as $10 \%$ by the expert and its corresponding matching degrees associated with the referential values (in this case they are "High", "Medium" and "Low") obtained by using equations (3), (4) and (5). A rule is said to be activated when the matching degrees $\left(\alpha_{H}, \alpha_{M}, \alpha_{L}\right)$ are assigned to one of the referential values of the antecedent attribute. This phenomenon is considered as the packet antecedent of a rule. The reason for assigning matching degree to only one referential value of the antecedent attribute of a rule is that only one referential value is considered as shown in Eq. (1). A belief rule base consists of a number of rules. However, the remaining matching degrees will be assigned to the corresponding referential values of an antecedent attribute by taking account of the fact that they are related to different belief rules.

TABLE II. INPUT TRANSFORMATION

\begin{tabular}{|l|l|l|l|l|l|}
\hline $\begin{array}{l}\text { Serial } \\
\text { No. }\end{array}$ & $\begin{array}{l}\text { Antecedent } \\
\text { Name }\end{array}$ & $\begin{array}{l}\text { Antecedent } \\
\text { Value }\end{array}$ & High & Medium & Low \\
\hline 1 & Rash & Low, $10 \%$ & 0.0 & 0.2 & 0.8 \\
\hline 2 & $\begin{array}{l}\text { Congested } \\
\text { Conjunctiva }\end{array}$ & $\begin{array}{l}\text { Medium, } \\
50 \%\end{array}$ & 0.0 & 1 & 0 \\
\hline 3 & Relative Bradicardia & High, $80 \%$ & 0.6 & 0.4 & 0 \\
\hline
\end{tabular}

2) Rule Activation Weight calculation

This consists of calculating the combined matching degree Rule followed by the calculation of its activation weight. Eq. (1) illustrates that the rule consists of more than one antecedent attributes. Therefore, the combined matching degree of a rule considering all the antecedent attributes is necessary to find out. By applying simple weighted multiplicative functions as shown in Eq. (6) this can be achieved. The use of this function allows the demonstration of the relationship among the antecedent attributes of a rule.

$$
\alpha_{k i}=\prod_{i=1}^{T_{k}}\left(\alpha_{i}^{k}\right)^{\delta_{k i}^{\prime}}
$$

Here, $\alpha_{i}$ represents the individual matching degree of a referential value of an antecedent attribute as described earlier. $\delta_{k t}$ is the relative weight of an antecedent attribute, which is used in the $k$ th rule and is calculated by dividing the weight of the attribute by the maximum weight of all antecedent attributes of the $k$ th rule to normalize the value of $\delta_{k t}$ which means that its value should range between 0 and 1 .

When the $k$ th rule is activated, the weight of activation of the $k$ th rule, $\omega k$, is calculated by using the following formula [17][18][19][20].

$$
\omega_{k}=\frac{\theta_{k} \alpha_{k}}{\sum_{j=1}^{L} \theta_{j} \alpha_{j}}=\frac{\theta_{k} \prod_{i=1}^{T_{k}}\left(\alpha_{i}^{k}\right)^{\delta_{k i}^{\prime}}}{\sum_{j=1}^{L} \theta_{j}\left[\prod_{i=1}^{T_{k}}\left(\alpha_{i}^{j}\right)^{\delta_{j i}^{\prime}}\right]}, \delta_{k i}^{\prime}=\frac{\delta_{k i}}{\max _{i=1, \ldots, T_{k}}\left\{\delta_{k i}\right\}}
$$

\section{3) Belief Update}

There could be some situations when it would not be possible to collect the input values of all the antecedent attributes of a rule and hence, the uncertainty due to ignorance occurs. Such situation of uncertainty can be handled by updating the belief degrees assigned to the referential values of the consequent attribute during the development of the initial belief rule base by applying Eq. (6).

$$
\beta_{i k}=\bar{\beta}_{i k} \frac{\sum_{t=1}^{T_{k}}\left(\tau(t, k) \sum_{j=1}^{J_{t}} \alpha_{t j}\right)}{\sum_{t=1}^{T_{k}} \tau(t, k)}
$$

where

$$
\tau(t, k)=\left\{\begin{array}{c}
1, \text { if } P_{i} \text { is used in defining } R_{k}\left(t=1, \ldots, T_{k}\right) \\
0, \text { otherwise }
\end{array}\right.
$$

Here, $\bar{\beta}_{i k}$ is the original belief degree while $\beta_{i k}$ is the updated belief degree. The original belief degree updated if any 
ignorance noticed. For example, if the antecedent "headache" is ignored, then the initial belief degrees are updated as shown in Table 3.

TABLE III. BELIEF DEGREE UpdATE

\begin{tabular}{|c|c|c|c|c|c|}
\hline \begin{tabular}{|l} 
Rule \\
Id \\
\end{tabular} & & \begin{tabular}{|l} 
High \\
D1
\end{tabular} & \begin{tabular}{|l|} 
Medium \\
D2
\end{tabular} & \begin{tabular}{|l} 
Low \\
D3
\end{tabular} & \begin{tabular}{|l|} 
Activation \\
Weight
\end{tabular} \\
\hline \multirow{3}{*}{1} & Initial & 1 & 0 & 0 & \multirow{3}{*}{0.096} \\
\hline & $\begin{array}{l}\text { Matching } \\
\text { Degree }\end{array}$ & 1 & 0 & 0 & \\
\hline & Update & 0.096 & 0 & 0 & \\
\hline \multirow{3}{*}{2} & Initial & 0.6 & 0.3 & 0.1 & \multirow{3}{*}{0.064} \\
\hline & $\begin{array}{l}\text { Matching } \\
\text { Degree }\end{array}$ & 0.7 & 0.2 & 0.1 & \\
\hline & Update & 0.0448 & 0.0128 & 0.0064 & \\
\hline
\end{tabular}

4) Rule Aggregation

In order to diagnose the dengue fever based on the input values of the signs, symptoms and risk factors, the activated rules need to be aggregated. The aggregation can be achieved by applying the Eq. (9), which encapsulates the evidential reasoning algorithm [4][11].

$$
\begin{gathered}
\beta_{j}=\frac{\mu \times\left\lfloor\prod_{k=1}^{L}\left(\omega_{k} \beta_{j k}+1-\omega_{k} \sum_{j=1}^{N} \beta_{j k}\right)-\prod_{k=1}^{L}\left(1-\omega_{k} \sum_{j=1}^{N} \beta_{j k}\right)\right]}{1-\mu \times\left[\prod_{k=1}^{L} 1-\omega_{k}\right]} \\
\mu=\left[\begin{array}{l}
\left.\sum_{j=1}^{N} \prod_{k=1}^{L}\left(\omega_{k} \beta_{j k}+1-\omega_{k} \sum_{j=1}^{N} \beta_{j k}\right)-(N-1)\right]^{-1} \\
\times \prod_{k=1}^{L}\left(1-\omega_{k} \sum_{j=1}^{N} \beta_{j k}\right)
\end{array}\right.
\end{gathered}
$$

When the diagnosis of the dengue fever achieved [using Eq. (8)] with certain belief degrees associated with each of the referential values (High, Medium, Low), this can be converted into crisp value by using some preference values [4] by applying Eq. (9).

$$
y_{m}=\sum_{n=1}^{N} D_{n} \times B_{n(m)}
$$

\section{Belief RUle BASED EXPERT SyStem (BRBES) TO DiAgNOSE DENGUE FEVER}

This section presents the design, implementation, knowledge base construction and an overview of BRBES interface.

\section{A. Architecture, Design and Implementation of the BRBES}

The architecture of a system can be defined as the organization of its components, consisting of inputs, process, and outputs. In addition, the pattern of the system organization, known as architectural style, is also considered. The proposed BRBES architectural framework adopts three-layer architectural style, consisting of interface, application processing and data management layers as depicted Fig. 2.
The explanation of this architecture is given below:

\section{1) Interface Layer}

This layer enables the interaction between the users and the BRBES. Users can input the value of the antecedent attribute as well as can see the system outputs. A combination of tools used to develop the BRBES interface, consisting of PHP, HTML, Javascript, and JQuery. The reason for using these tools is that they allow the creating of user-friendly web-based interface by using simple code.

\section{2) Application processing layer}

This layer supports the inference procedures of the BRBES consisting of input transformation, rule activation weight calculation, belief update and the rule aggregation. PHP has been used in this layer since due to its simplicity and shorter development cycles this language is largely used. Moreover, it allows the system to be accessible through online.

\section{3) Data Management Layer}

This layer stores and manages the knowledge base. MySQL, which is a flexible and user-friendly relational database, is used at the back-end to store and manipulate the initial BRB. MySQL also allows the faster access of data and ensures the security as needed by the BRBES.

There exist some individuals such as domain experts, knowledge engineers and users of the system, who play important roles to acquire knowledge and to develop the knowledge base of the expert system. Domain experts who have the knowledge of dengue fever and in our case they are the physicians engaged in giving treatment to the patients. On the contrary, knowledge engineers encode the expert's knowledge in a declarative form that can be used by the expert system in the form of initial belief rule-base. The BRBES architecture as shown in Fig. 1 incorporates the above viewpoint.

\section{B. Knowledge Base Construction in BRB}

In consultation with the domain experts a BRB framework to diagnose dengue fever has been developed (Fig. 2) and this is considered as the starting point to construct the knowledge base. The BRB framework as depicted in Fig. 2 represents a hierarchical multilevel as well as nonlinear structure necessary to diagnose the dengue fever. The tree consists of three different types of nodes namely leaf nodes, mid-level nodes and the root node. The data related to the leaf nodes attributes need to be collected to obtain the root node value and this can be obtained by applying various inference algorithms as mentioned before.

For example, the level of the critical signs (A11) of the dengue fever of a patient can be assessed by getting input values of the three child nodes (A4, A5, and A6). 


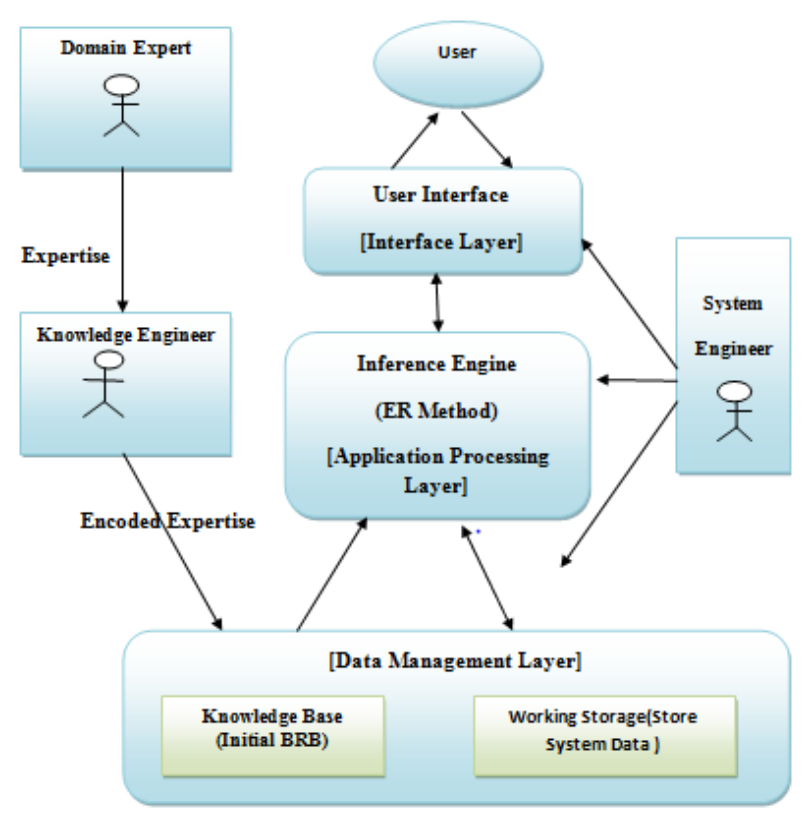

Fig. 1. The BRBES architecture

The BRB framework to diagnose dengue fever consist of four sub-rule bases namely A13, A12, A11 and A10. The total number of rules in a sub-rule base can be calculated by the following Eq. (11).

$$
L=\prod_{i=1}^{T} J_{i}
$$

where

$L=$ Total number of rules in a sub rule base

$J_{i}=$ Referential values of $\mathrm{i}^{\text {th }}$ antecedent attributes

For example, A11 sub rule base consists of three attributes (A4, A5, and A6) and each of them with three referential values. Hence, $\mathrm{J} 4=3, \mathrm{~J} 5=3$, and $\mathrm{J} 6=3$, and therefore $\mathrm{L}=(3 * 3 * 3)=27$ after applying Eq. (11). Thus, this sub rule base consists of 27 rules as illustrated in Table 4. A BRB can be established in four different ways: (1) by using domain expert knowledge; (2) by examining historical data; (3) by using previous rules if available; and (4) by creating rules in a random way without any prior knowledge. In this research, initial BRB has been constructed by extracting heuristic belief rules from the domain experts. It is assumed that all the rules and the attributes have equal weight. Initial BRB for the A11 sub rule base is listed in Table 4. An example of a belief rule taken from Table 4 is elaborated below.

R6: IF Rash is High AND Congested Conjunctivas is Medium AND Relative Bradicardia is Low THEN Critical Sign is $\{($ High,0), (Medium, 0.5), (Low,0.5)\}.

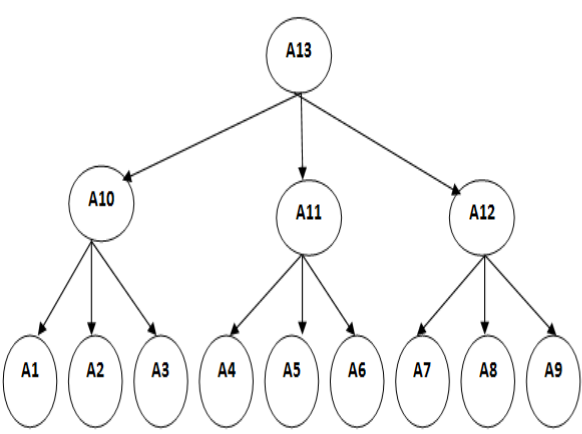

\begin{tabular}{l} 
A1: Fever \\
A2: Headache \\
A3: Body ache \\
A4: Rash \\
A5: Congested \\
conjunctiva \\
A6: Relative Bradicardia \\
A7: Population \\
A8: Mosquito breeding \\
place \\
A9: Mosquito bite \\
A10: Common Sign \\
A11: Critical Sign \\
A12: Risk Factor \\
A13: Dengue \\
\hline
\end{tabular}

Fig. 2. The BRB Framework to Diagnose Dengue Fever

TABLE IV. INITIAL BRB FOR A11 Sub RULE BASE

\begin{tabular}{|c|c|c|c|}
\hline \multirow{2}{*}{$\begin{array}{l}\text { Rule } \\
\text { Id }\end{array}$} & \multirow{2}{*}{$\begin{array}{l}\text { Rule } \\
\text { weight }\end{array}$} & IF Antecedent & THEN Consequent \\
\hline & & $\mathrm{A4} \wedge \mathrm{A} 5 \wedge \mathrm{A6}$ is & A11 is \\
\hline 1 & 1 & $\mathrm{H} \wedge \mathrm{H} \wedge \mathrm{H}$ & $\{(\mathrm{H}, 1.00),(\mathrm{M}, 0.00),(\mathrm{L}, 0.00)\}$ \\
\hline 2 & 1 & $\mathrm{H} \wedge \mathrm{H} \wedge \mathrm{M}$ & $\{(\mathrm{H}, 1.00),(\mathrm{M}, 0.00),(\mathrm{L}, 0.00)\}$ \\
\hline 3 & 1 & $\mathrm{H} \wedge \mathrm{H} \wedge \mathrm{L}$ & $\{(\mathrm{H}, 0.9),(\mathrm{M}, 0.1),(\mathrm{L}, 0.00)\}$ \\
\hline 4 & 1 & $\mathrm{H} \wedge \mathrm{M} \wedge \mathrm{H}$ & $\{(\mathrm{H}, 0.4),(\mathrm{M}, 0.5),(\mathrm{L}, 0.1)\}$ \\
\hline 5 & 1 & $\mathrm{H} \wedge \mathrm{M} \wedge \mathrm{M}$ & $\{(\mathrm{H}, 0.3),(\mathrm{M}, 0.4),(\mathrm{L}, 0.3)\}$ \\
\hline 6 & 1 & $\mathrm{H} \wedge \mathrm{M} \wedge \mathrm{L}$ & $\{(\mathrm{H}, 0.00),(\mathrm{M}, 0.5),(\mathrm{L}, 0.5)\}$ \\
\hline$\ldots$ & $\ldots$. & $\ldots$ & $\ldots$. \\
\hline 25 & 1 & $\mathrm{~L} \wedge \mathrm{L} \wedge \mathrm{H}$ & $\{(\mathrm{H}, 0.00),(\mathrm{M}, 0.0),(\mathrm{L}, 1.00)\}$ \\
\hline 26 & 1 & $\mathrm{~L} \wedge \mathrm{L} \wedge \mathrm{M}$ & $\{(\mathrm{H}, 0.00),(\mathrm{M}, 0.00),(\mathrm{L}, 1.00)\}$ \\
\hline 27 & 1 & $\mathrm{~L} \wedge \mathrm{L} \wedge \mathrm{L}$ & $\{(\mathrm{H}, 0.00),(\mathrm{M}, 0.00),(\mathrm{L}, 1.00)\}$ \\
\hline
\end{tabular}

\section{BRB Interface}

An interface is a media through which the users interact with the system. Fig. 3 illustrates the interface of the BRBES to diagnose dengue fever. The interface allows the inputting of the leaf nodes data of the BRB framework (Fig. 3), which are collected from the patients or the physicians. This interface facilitates the displaying of the results not only at the top level but also at the mid-level in terms of fuzzy values or crisp values (Fig. 3). These fuzzy values can be converted into crisp value by using Eq. 10. For example, the fuzzy values of the "common sign", which is a mid-level node, obtained for its different referential values consists of (High, 75.09\%), (Medium, 22.688\%) and (Low, 2.222\%). The belief degree attached with the referential values of the "common sign" calculated by using Eq. (9). Using Eq. (10) the crisp value for these fuzzy values obtained which is 86.434 . In this way, the final crisp value for the top-level node or the root node can be obtained which is $71.433 \%$ (Fig 3 ).

\section{RESULTS AND DISCUSSION}

To test the reliability and the validity of the BRBES to diagnose dengue fever the leaf nodes data of the BRB tree (Fig. 2) from the patients of the two hospitals, located in the city of 
Chittagong, Bangladesh, collected. The collected data were fed into BRBES to obtain the suspicion level of dengue fever for each patient as shown in Table V. In addition, the expert opinion or the opinion of the concerned physicians on the suspicion level of dengue fever of each the patient was also collected as shown in Table V.

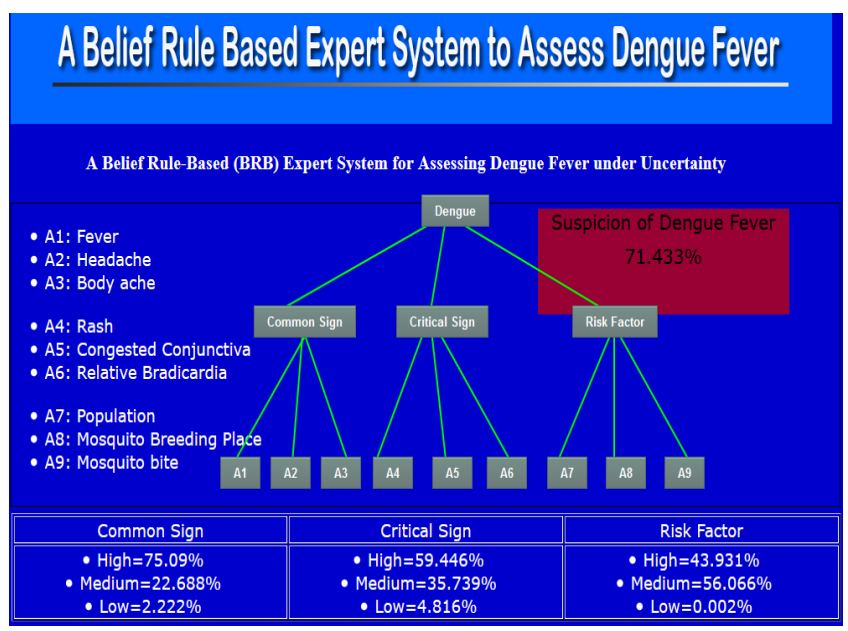

Fig. 3. BRBES Interface for Dengue Fever

It is interesting to note that the physicians were not aware of the uncertainty phenomenon associated with the signs and symptoms of the dengue fever. Eventually, there could be high risk of inaccuracy with the physicians' suspicion level of dengue fever. Laboratory test data of the patients considered as the benchmark data. For example, if the lab test confirms a patient with dengue fever then it is considered as " 1 " otherwise it is considered as " 0 ". 100 dataset have been collected from the patients to test the BRBES. For simplicity, Table V only presents data collected from ten patients.

The Receiver Operating Characteristic (ROC) curves are widely used to analyze the effectiveness of diagnostic tests having ordinal or continuous results [21][22]. Therefore, it has been considered to measure the reliability of BRBES. The accuracy or the performance of the BRBES in assessing the suspicion level of dengue fever can be measured by calculating the Area Under Curve (AUC). If the AUC is one then the results generated by the BRBES can be considered as accurate. The ROC curve plotted by the blue line in Fig. 4 is associated with the results generated by the BRBES with AUC of 0.833 (95\% confidence intervals $0.755-0.911)$. The ROC curve plotted by the green line in Fig. 4 is obtained against the physician's opinion, and its AUC is 0.810 (95\% confidence intervals $0.726-0.894$ ).

Table VI illustrates the results generated by using Fuzzy Logic Based Expert System (FLBES) along with BRBES and expert opinion for the same set of data as shown in Table V.

Fig. 5 illustrates the ROC curves for the BRBES, FLBES and expert opinion. The ROC curve plotted by the green line in Fig. 4 is associated with the results generated by the FLBES with AUC of 0.814 (95\% confidence intervals $0.730-0.898)$, which is less than AUC of BRBES (0.833).

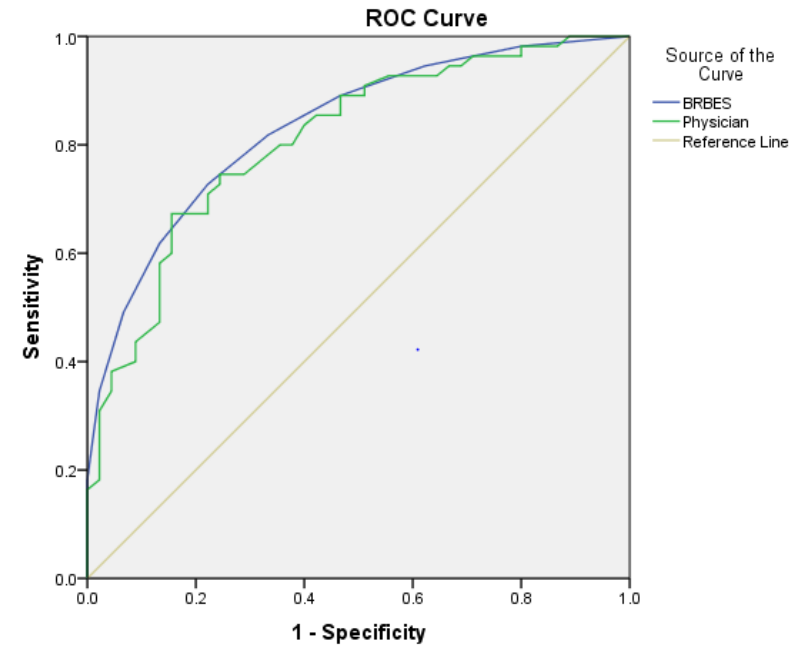

Fig. 4. ROC curves comparing BRBES's result and Expert's opinion

TABLE V. DENGUE AsSESSMENT RESUlt by BRBES AND FuZZY Logid BASED EXPERT (FRLES) SYSTEM

\begin{tabular}{|l|c|l|l|c|}
\hline $\begin{array}{l}\text { Case } \\
\text { Study }\end{array}$ & BRBES & $\begin{array}{l}\text { Expert } \\
\text { Opinion }\end{array}$ & FLBES & Output \\
\hline 1 & 65.4440 & 65 & 63.9 & 1 \\
\hline 2 & 84.6215 & 85 & 76.8 & 1 \\
\hline 3 & 37.9265 & 39 & 42.9 & 0 \\
\hline 4 & 81.9800 & 83 & 75.3 & 1 \\
\hline 5 & 59.3295 & 53 & 59.4 & 0 \\
\hline 6 & 71.4330 & 70 & 68 & 1 \\
\hline 7 & 75.5745 & 70 & 74.5 & 1 \\
\hline 8 & 44.8850 & 42 & 37 & 0 \\
\hline 9 & 29.3610 & 25 & 29 & 0 \\
\hline 10 & 51.2925 & 55 & 49.6 & 0 \\
\hline
\end{tabular}

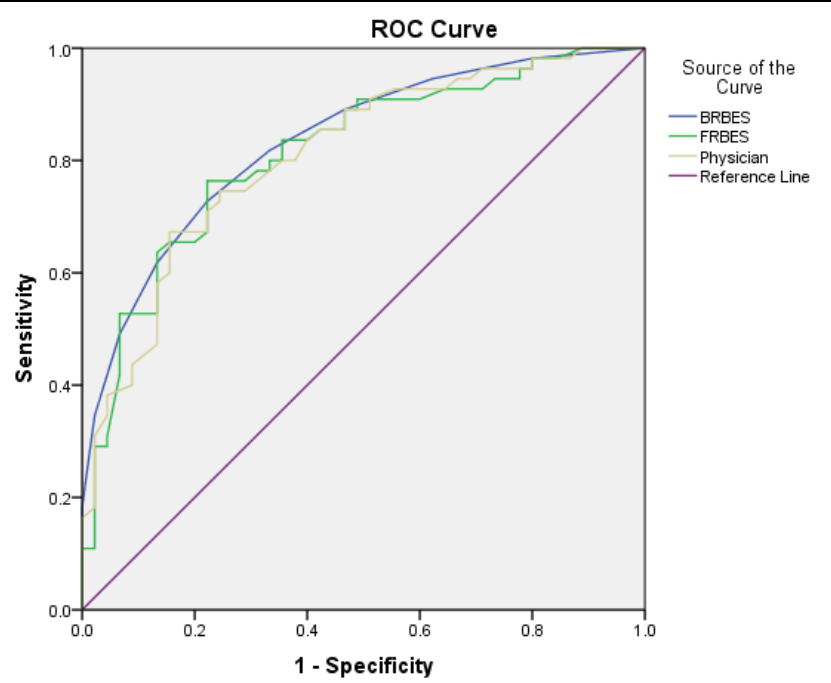

Fig. 5. ROC curves for comparing BRBES and Fuzzy Rule Based Expert System (FRBES) 
TABLE VI. DATASET For System TESTING

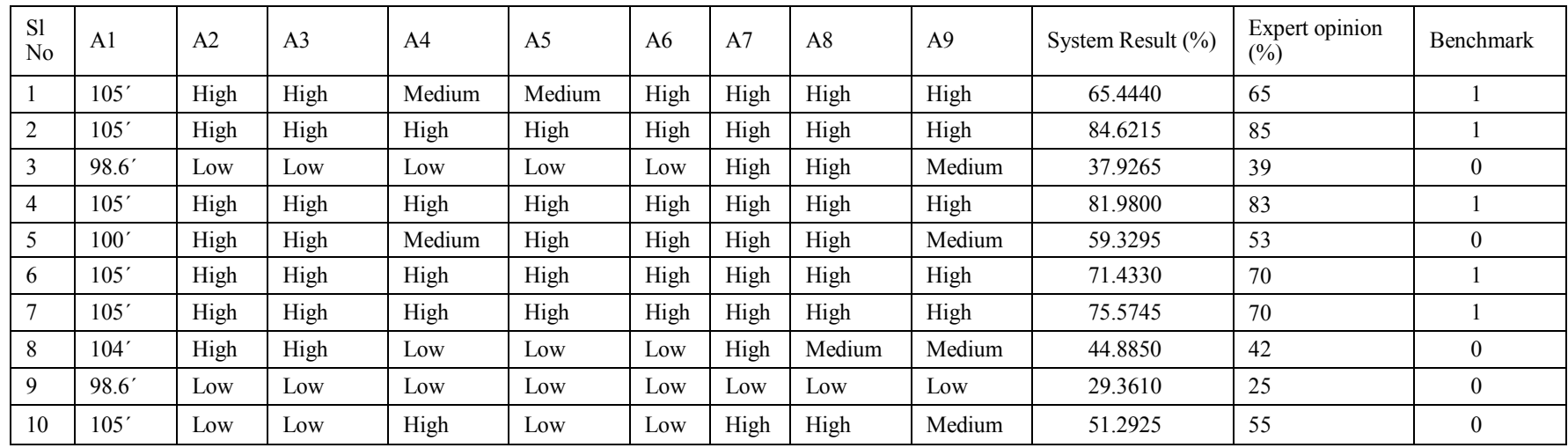

TABLE VII. RELIABILITY COMPARISON AMONG THREE EXPERT SySTEMS

\begin{tabular}{|l|l|l|}
\hline Expert System & AUC & $\begin{array}{l}\text { Asymptotic 95\% } \\
\text { confidence interval }\end{array}$ \\
\hline BRBES & 0.833 & $0.755-0.911$ \\
\hline FLBES & 0.814 & $0.730-0.898$ \\
\hline Expert's opinion & 0.810 & $0.726-0.894$ \\
\hline
\end{tabular}

\section{CONCLUSION}

In recent years, the fatality rate of dengue fever increases substantially all over the world. Therefore, an effective system to diagnose dengue disease is essential for the people who are living in dengue risk zones of the world. This research demonstrates the development and applications of a belief rule based expert system (BRBES) with the ability to handle various types of uncertainties associated with the signs, symptoms and risk factors of dengue fever. It has been demonstrated that the BRBES generated results are better than that of expert opinion or the fuzzy rule based expert system. In addition, different types of scenarios or the simulated results can be generated by using the BRBES, for example at the midlevel factors of the BRBES tree such as "Common Sign", "Critical sign" and "risk factors". This type of capability will help to analysis the disease from various perspectives to develop an appropriate strategic plan to reduce the risk of dengue fever in the context of a country.

\section{ACKNOWLEDGMENT}

We would like to extend our gratitude to the Swedish Research Council (under grant 2014-4251) for the funding and the provision of resources to conduct this research.

\section{REFERENCES}

[1] World Health Organization, "Dengue and dengue haemorrhagic fever," Factsheet No 117, revised May 2008. Geneva, World Health Organization, 2008 (http: //www. who. int /mediacentre/ factsheets/fs117/en/).

[2] F.P. Pinheiro and S.J. Corber, "Global situation of dengue and dengue haemorrhagic fever and its emergence in the Americas," World Health Stat Q 1997;50:161-169.

[3] World Health Organisation, "Prevention and control of dengue and dengue haemorrhagic fever: comprehensive guidelines," WHO Regional publication, SEARO, No 29, 1999.

[4] Alameda County Mosquito Abatement District, "Biological Notes on Mosquitoes," Life cycle of the mosquito (2011).
[5] G. Kuno, "Factors Influencing the Transmission of Dengue Viruses, "In Dengue and Dengue Hemorrhagic Fever," eds. D. J. Gubler \& G. Kuno (Cambridge:CABI, 2001):61-88.

[6] F. Rodhain and L. Rosen, "Mosquito Vectors and Dengue Virus-Vector Relationships." In Dengue and Dengue Hemorrhagic Fever, eds. D. J. Gubler \& G. Kuno (Cambridge: CABI, 2001):45-60

[7] World Health Organization, "Dengue: Guidelines for Diagnosis, Treatment, Prevention and Control," Geneva: World Health Organization and the Special Programme for Research and Training in Tropical Diseases, 2009.

[8] C.H. Kuo, D.I. Tai, C.S. Chang-Chein, C.K. Lan, S.S. Chiou, and Y.F. Liaw, "Liver Biochemical test and Dengue Fever", The American Journal of Tropical Medicine and Hygiene, 47(3):265-270, 1992.

[9] F. Ibrahim, M.N. Taib., W.A.B.W. Abas, C.C. Guan, and S. Sulaiman, "A novel approach to classify risk in dengue hemorrhagic fever (DHF) using bioelectrical impedance analysis," IEEE Transactions on Instrumentation and Measurement 54(1):237-244, 2005.

[10] D.E. Carey, "Chikungunya and dengue: A case of mistaken identity?" J. Hist. Med. Allied Sci. 26(3):243-262, 1971.

[11] P.M. Prihatini and K.G.D. Putra, "Fuzzy Knowledge-based System with Uncertainty for Tropical Infectious Disease Diagnosis," IJCS International Journal of Computer Science Issues, Vol. 9, Issue 4, No 3, pp. 157-163, 2012.

[12] F. Ibrahim, M.N. Taib, W.A. Abas, C.C. Guan, and S. Sulaiman, "A novel dengue fever (DF) and dengue haemorrhagic fever (DHF) analysis using artificial neural network (ANN)," Computer Methods Programs Biomed. 79(3):273-281, 2005.

[13] F. Ibrahim, M.I. Mohamad, S.N. Makhtar and J. Ibrahim, "Classification of Risk in Dengue Fever and dengue Haemorrhagic Fever using Rule Based Expert System," 3rd Kuala Lumpur International Conference on Biomedical Engineering, pp. 50-53, 2006.

[14] S. Karim, H. Suryaningsih, and A. Lause, "Expert System for Diagnosing Dengue Fever", Seminar Nasional Aplikasi Teknologi Informasi (SNATI 2007)

[15] M.S. Hossain, K. Andersson, and S. Naznin, "A Belief Rule Based Expert System to Diagnose Measles under Uncertainty," 2015 International Conference on Health Informatics and Medical Systems (HIMS'15), Las Vegas, NV, USA, 2015.

[16] R. Karim, K. Andersson, M.S. Hossain, M.J. Uddin, and M.P. Meah, “A Belief Rule based Expert System to Assess Clinical Bronchopneumonia Suspicion," Future Technologies Conference (FTC 2016), San Francisco, CA, USA, 2016.

[17] J.B. Yang, J. Liu, J.Wang, H.S. Sii and H.W. Wang, "Belief rule-base inference methodology using the evidential reasoning approachRIMER", IEEE Transactions on Systems Man and Cybernetics Part ASystems and Humans 36(2):266-285, 2006.

[18] J.B. Yang, "Rule and utility based evidential reasoning approach for multi-attribute decision analysis under uncertainties", European Journal of Operational Research 131(1):31-61, 2007. 
[19] M.S. Hossain, P.O. Zander, S. Kamal, and L. Chowdhury, "Belief Rule Based Expert Systems to Evaluate E-Government: A Case Study," Expert Systems 32(5):563-577, 2015.

[20] Y.M. Wang, J.B. Yang, and D.L. Xu, "Environmental impact assessment using the evidential reasoning approach," European Journal of Operational Research 174(3):1885-1913, 2006.
[21] C.E. Metz. "Basic principles of ROC analysis," Semin. Nucl. Med. 8(4):283-298, 1978.

[22] J.A. Hanley. "The Robustness of the "Binormal," Assumptions Used in Fitting ROC Curves,” Med. Decis. Making 8(3):197-203, 1988. 